Research Paper

\title{
Disregulations of PURPL and MiR-338-3p Could Serve As Prognosis Biomarkers for Epithelial Ovarian Cancer
}

\author{
Ruitao Zhang\# ${ }^{\bowtie}$, Tingting He\#, Huirong Shi, Cao Yuan, Feng Wei, Zheying Liu, Wenwen Wang \\ Department of Gynecology, First Affiliated Hospital, Zhengzhou University, NO.1 East Jianshe Road, Erqi District, Zhengzhou, Henan, 450052 P.R. China \\ \#These authors contributed equally to this study and share first authroship \\ $\bowtie$ Corresponding author: Ruitao Zhang: fcczhangrt@zzu.edu.cn
}

(c) The author(s). This is an open access article distributed under the terms of the Creative Commons Attribution License (https://creativecommons.org/licenses/by/4.0/). See http://ivyspring.com/terms for full terms and conditions.

Received: 2021.04.06; Accepted: 2021.07.19; Published: 2021.07.25

\begin{abstract}
Objective: The present study aimed to explore the expressions of long noncoding RNA (IncRNA) p53 upregulated regulator of $\mathrm{P} 53$ levels (PURPL) in different ovarian tissues, and to evaluate the significance of disregulations of PURPL and microRNA-338-3p (miR-338-3p) in epithelial ovarian cancer (EOC).

Methods: The expressions of PURPL in ovarian cancer, the relations between PURPL and the prognosis of ovarian cancer, and the relation between PURPL and miR-338-3p were queried in multiple biomedical databases. Real-time PCR was performed to detect the expressions of PURPL in different ovarian tissues. Logistic regression analysis was used to analyze the risk factors of recurrence and death. Kaplan-Meier analysis was implemented to evaluate the relations between PURPL and miR-338-3p expressions and the survival of ovarian cancer.

Results: PURPL could target miR-338-3p, PURPL were upregulated in ovarian cancer tissues, upregulation of PURPL in ovarian cancer was negatively related with the recurrence free survival (RFS) and overall survival (OS), which were indicated by biomedical databases query. Our data showed upregulations of PURPL were noted in ovarian cancer tissues. Higher expressions of PURPL were associated with more advanced FIGO stage and developed lymph node metastasis in epithelial ovarian cancer. Upregulation of PURPL was related with the recurrence $(P=0.002, O R=21.482,95 \% \mathrm{Cl}$ : 3.457 94.251) and death ( $P=0.004, O R=35.643,95 \% \mathrm{Cl}: 2.453 \sim 84.359)$ of ovarian cancer patient. PURPL expressions were negatively correlated to miR-338-3p expressions in different ovarian tissues $(r=$ $-0.968, P<0.0001)$. Poor RFS $\left(\chi^{2}=19.410, P=0.0002\right)$ and OS $\left(\chi^{2}=17.600, P=0.0005\right)$ were found in patients with high level PURPL and low level miR-338-3p expressions.

Conclusions: Upregulation of PURPL and downregulation of miR-338-3p were related with the poor RFS and OS of ovarian cancer, which indicated disregulations of PURPL and miR-338-3p could serve as prognosis biomarkers for epithelial ovarian cancer.
\end{abstract}

Key words: biomedical database; epithelial ovarian cancer; PURPL; miR-338-3p; prognosis

\section{Introduction}

Although gene target therapy and immunity therapy bring new hope to the treatments of ovarian cancer patients, ovarian cancer still is the most lethal malignancy in female genital tract tumors [1]. Even though considerable progress in molecular mechanism research of the growth and metastasis of ovarian cancer has been made, further research is still needed. Emerging reports have shown that noncoding RNAs, including long noncoding RNAs
(lncRNAs) and micro noncoding RNAs (miRNAs), play important roles in the growth and metastasis of human malignant tumors [2-6]. LncRNAs were reported to be important regulatory molecules that could regulate the growth and metastasis of malignant tumors via targeting and sponging downstream miRNAs [7-8].

Our previous data indicated miR-338-3p was downregulated and MACC1 was upregulated in 
epithelial ovarian cancer, and was related with poor prognosis of ovarian cancer [9]. We tried to explore the upstream regulated lncRNAs of miR-338-3p to analyze their roles in the development and progression of ovarian cancer in present study. Biomedical database queries showed that lncRNA p53 upregulated regulator of p53 levels (PURPL) could target miR-338-3p. PURPL, also known as LINC01021, was detected to be upregulated in gastric cancer tissue, and was related to tumor size and histopathological grade, which indicated poor clinical outcomes [10]. Rare reports were involved in the relations between PURPL and ovarian cancer.

In the present study, we tried to query the expression of PURPL and its relation with the prognosis of ovarian cancer in biomedical databases. Then, real time PCR was performed to confirm the expressions of PURPL in different collected ovarian tissues, and to explore the significance of PURPL and $\mathrm{miR}-338-3 \mathrm{p}$ in the prognosis of ovarian cancer.

\section{Methods}

\section{Biomedical database query}

Multiple biomedical databases, including ENCORI [11], miRcode (http://www.mircode.org/) and LncBase Predicted [12] were used to predict the relation between PURPL and miR-338-3p. Pubmed (https:/ / pubmed.ncbi.nlm.nih.gov/), cBioPortal [13], IncRNASNP2 [14], GEPIA [15] were used to query the expressions of PURPL in normal and malignant human tissues. Kaplan-Meier Plotter [16] was used to analyze the relations between PURPL and the prognosis of ovarian cancer.

\section{Tissue specimens}

All enrolled fresh different ovarian tissue specimens of 105 patients were collected and saved in the First Affiliated Hospital of Zhengzhou University. There were 20 cases of normal ovarian tissues, 20 cases of benign ovarian epithelial tumor tissues, and 65 cases of primary lesions of newly diagnosed epithelial ovarian cancer. Complete detailed clinic parameters and detailed criteria for the inclusion and exclusion of patients enrolled were described in detail in our previous report [9]. Consent from patients or family members and approval by the Ethics Committee of the First Affiliated Hospital of Zhengzhou University was received for collection and use of all tissue samples.

\section{Real time PCR}

Total RNA isolation, cDNA reverse-synthesis and SYBR Green PCR assay procedures were described in our previous report [9]. The primers used in PCR were synthesized by Shanghai Sangon Biotech
Co., Ltd. The primer sequences were shown in Table 1. Each PCR experiment was performed three times independently, and the relative expression values were expressed by $2^{-\Delta \Delta C t}$ method.

Table 1. Primer sequences for real time PCR

\begin{tabular}{lll}
\hline Item & Sequences $\left(5^{\prime}\right.$ to $\left.3^{\prime}\right)$ & Product length $(\mathrm{bp})$ \\
\hline PURPL & $\begin{array}{l}\text { Forward:ACACGGGGCTTGAGAAATGA } \\
\text { Reverse:TCAATCTCCAAAATAGCCGGA }\end{array}$ & 376 \\
\multirow{2}{*}{-actin } & $\begin{array}{l}\text { Forward: CATGTACGTTGCTATCCAGGC } \\
\text { Reverse: CTCCTTAATGTCACGCACGAT }\end{array}$ & 250 \\
\hline
\end{tabular}

\section{Statistical Analysis}

Average values were expressed as mean \pm standard deviation, and GraphPad Prism 7 software package was used to analyze data. One-way ANOVA, Non-parametric test, Independent sample $\mathrm{T}$ test, Binary logistic regression analysis, Spearman correlation test and Kaplan-Meier analysis were performed respectively to explore the expression difference, relations with clinicopathologic characteristics, risk factors of recurrence and death analysis, expression interrelationship analysis, and survival analysis. Difference was considered significant when $P$ value was less than 0.05 .

\section{Results}

\section{Biomedical database query}

Based on our previous miR-338-3p data, multiple biomedical database cross-queries showed PURPL could target miR-338-3p (Figure 1). Further queries suggested that rare expressions of PURPL were detected in normal ovarian tissues in 27 types different normal human tissue samples from 95 human individuals (Figure 2A). In 32 TCGA PanCancer Atlas studies including 10967 samples, amplifications of PURPL were detected in several malignant human tumor tissues including ovarian serous cystadenocarcinoma tissues (Figure 2B). Compared to paired normal ovarian samples, upregulated profiles of PURPL were detected in ovarian carcinoma tissues among human malignant tumors (Figure 2B and 2B).

Compared to patients with low expression of PURPL, patients with high expression of PURPL showed shorter recurrence free survival (RFS) time and overall survival (OS) time, which was queried in Kaplan-Meier Plotter Pan-Cancer database including 374 ovarian cancer patients (Figure 3). Based on three TCGA databases including 1680 samples from 1668 ovarian cancer patients, we noted a negative expression profile between PURPL and miR-338-3p in ovarian cancer tissues (Figure 4). 
A

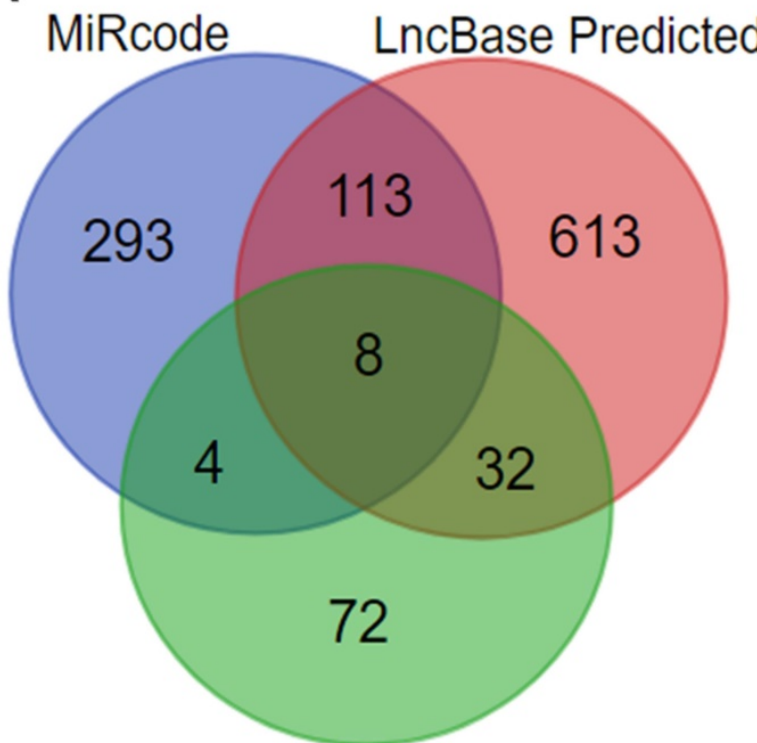

ENCORI
B

\begin{tabular}{|l|l|l|}
\hline List names & number of elements & number of unique elements \\
\hline ENCORI & 133 & 116 \\
\hline LncBase Predicted & 1043184 & 766 \\
\hline MiRcode & 418 & 418 \\
\hline Overall number of unique elements & $\mathbf{1 1 3 5}$ \\
\hline
\end{tabular}

\begin{tabular}{|l|l|l|}
\hline Names & total & elements \\
\hline \begin{tabular}{l|l|l|}
\hline ENCORI LncBase \\
Predicted \\
MiRcode
\end{tabular} & 8 & ENSC000000185004 ENSG000000224189 ENSG0000002058255 ENSG000000233086 \\
\hline
\end{tabular}

$\mathrm{C}$

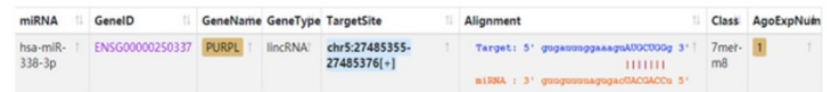

Figure 1. Biomedical database query predicted candidate IncRNAs targeted miR-338-3p (A.ENCORI, miRcode and LncBase Predicted databases crossed query Venn diagram for candidate IncRNAs targeted miR-338-3p; B. Input information and output result of Venn diagram producer showed PURPL (ENSG00000250337) was one of eight candidate IncRNAs targeted miR-338-3p; C. Targeted sites between PURPL and miR-338-3p indicated by ENCORI database.)

A

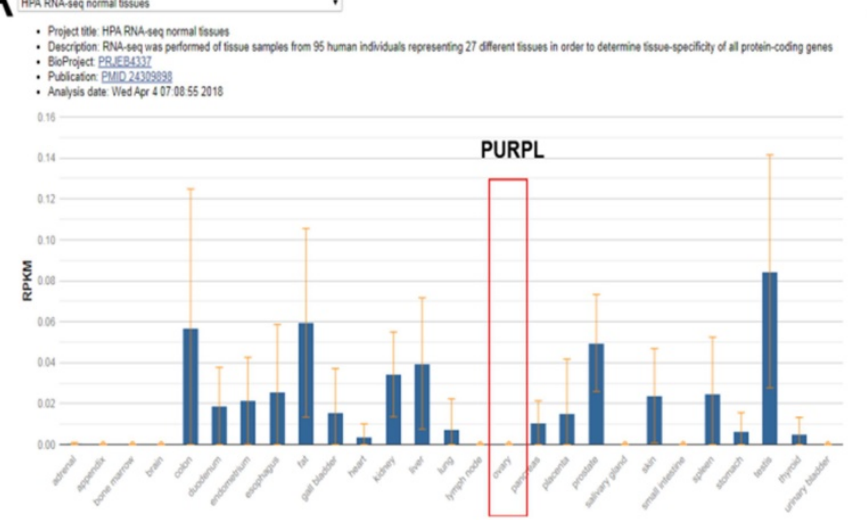

C

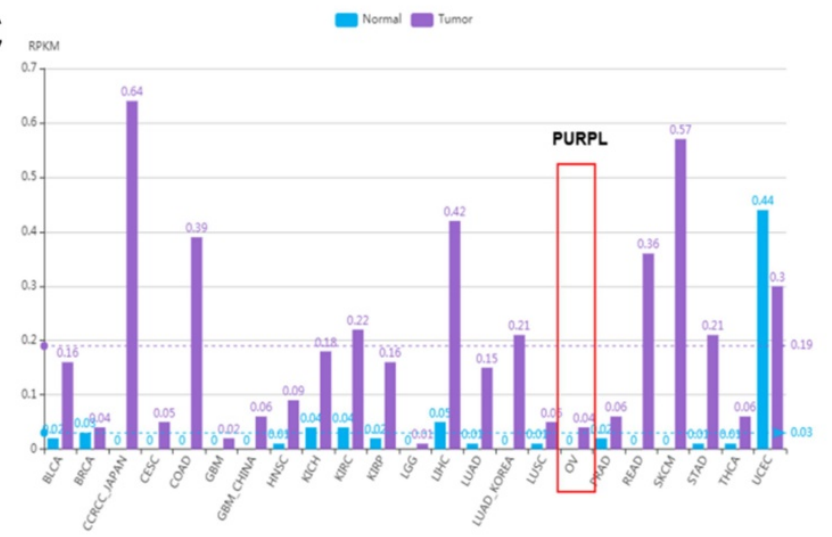

B
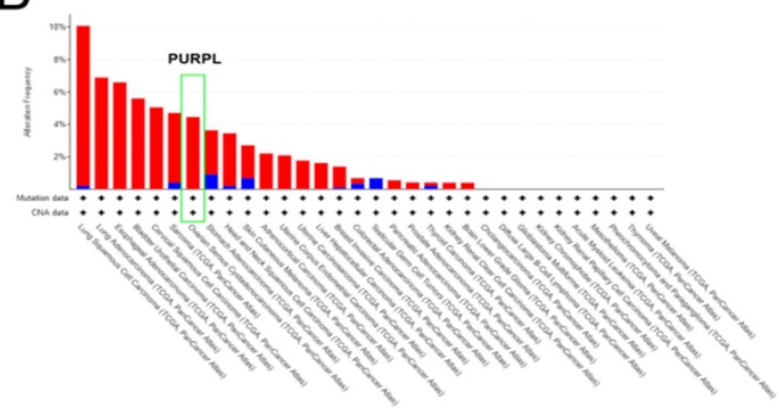

The gene expression profile across all tumor samples and paired normal tissues. (Dot plot)

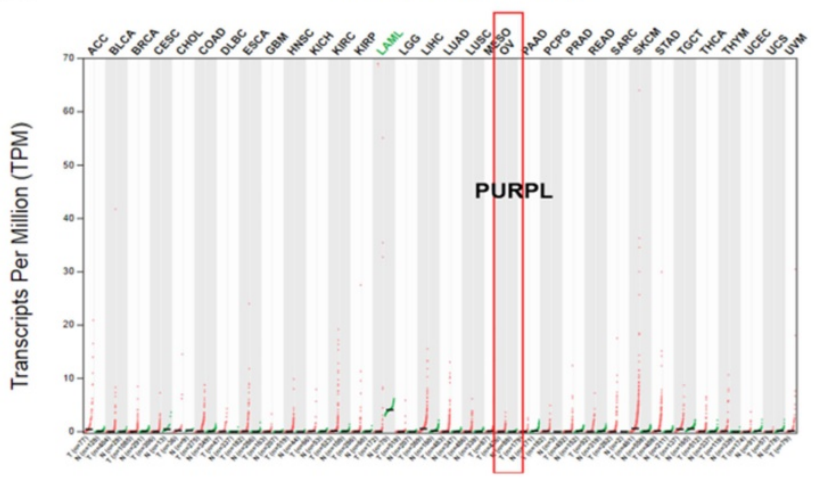

Figure 2. Expressions of PURPL in different human tissues queried from biomedical databases (A. Expressions of PURPL in different normal human tissues from Pubmed database; B. Expressions of PURPL in different malignant human tissues from cBioPortal database; C, D. Expressions of PURPL in paired normal ovarian and ovarian cancer tissues from IncRNASNP2 and GEPIA databases.) 
A

PURPL RFS

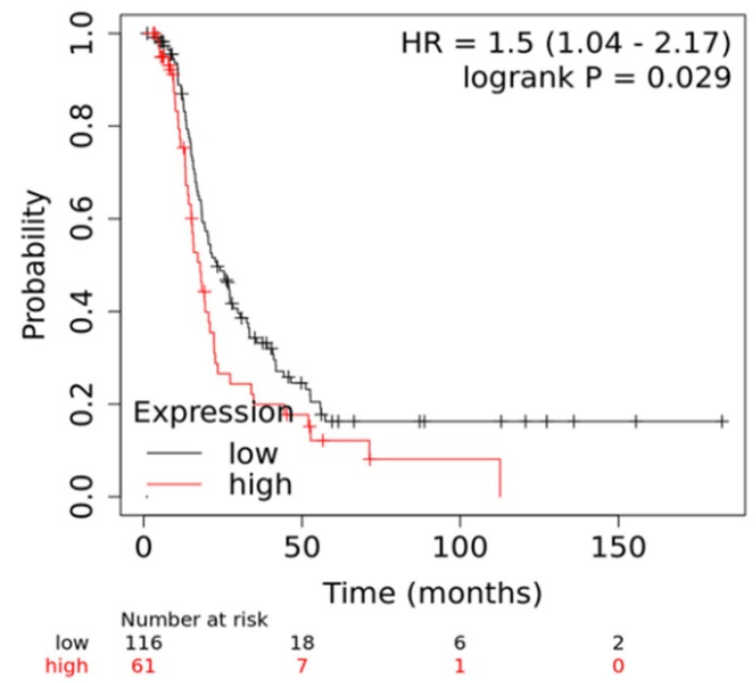

B

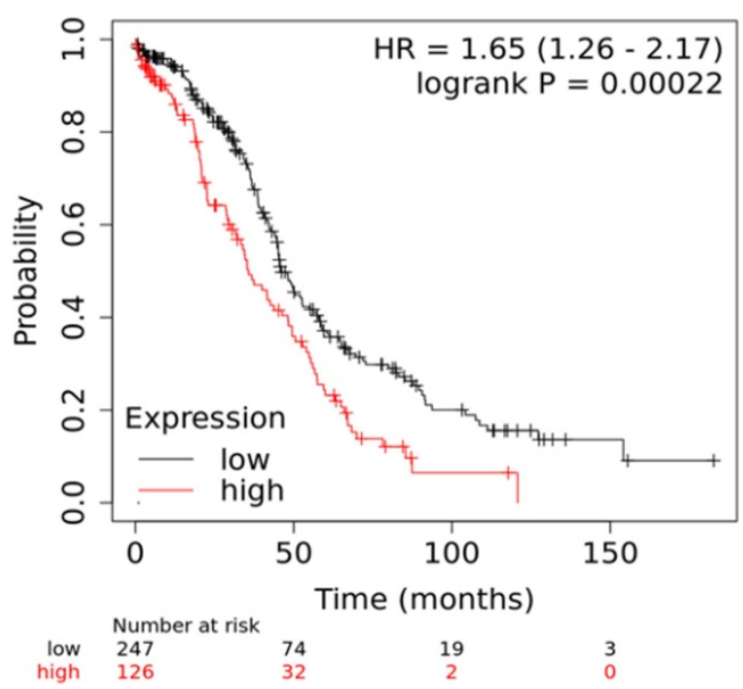

Figure 3. Relations of PURPL expression with the RFS and OS time of ovarian cancer from Kaplan Meier plotter database (A. RFS: recurrence free survival; B. OS: overall survival)

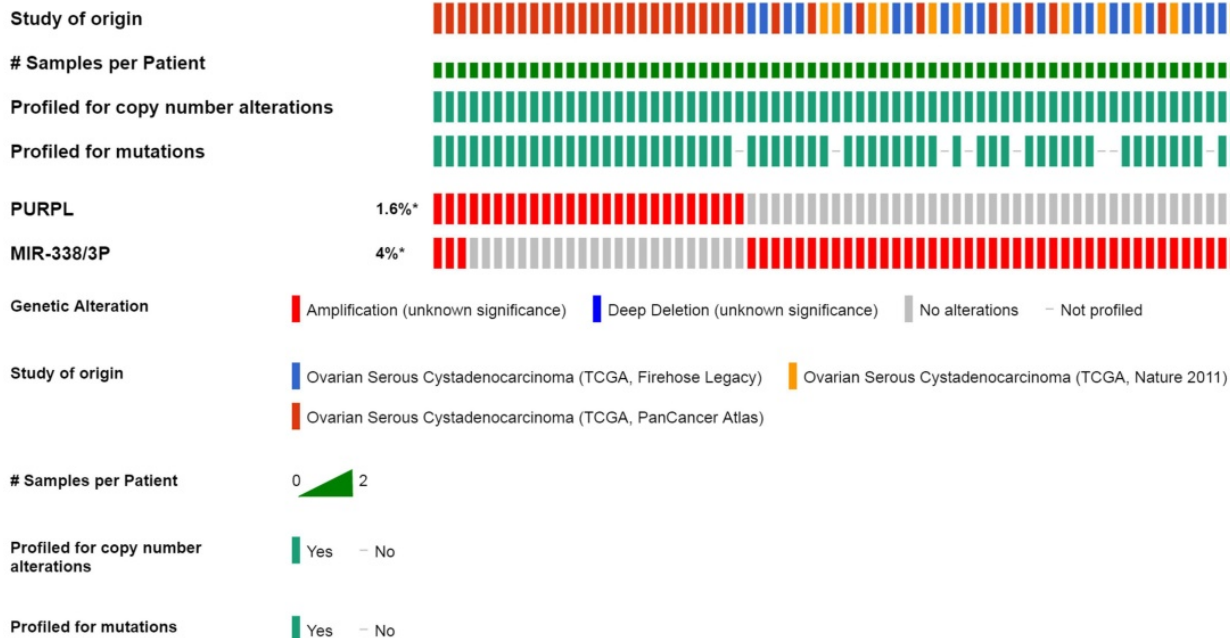

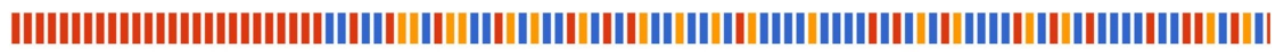

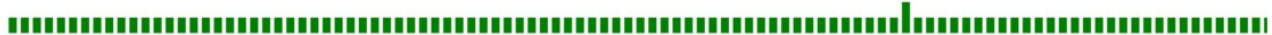

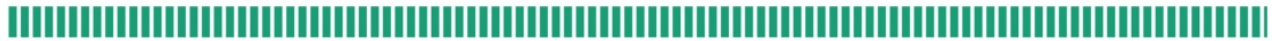

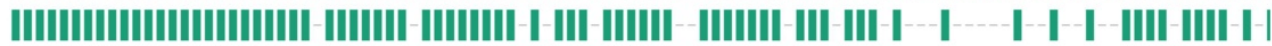

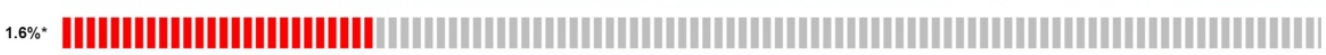
4\%*

Figure 4. Expression profile between PURPL and miR-338-3p in ovarian cancer tissues from cBioPortal database

\section{Expressions of PURPL in different collected ovarian tissues}

To verify the biomedical database query results, we collected normal ovarian, benign ovarian epithelial tumor and epithelial ovarian cancer tissues to analyze the expression profile of PURPL. Low expressions of PURPL were detected in normal ovarian and benign ovarian epithelial tumor tissues. However, relative expression level of PURPL in 65 cases ovarian cancer tissues was $(0.522 \pm 0.004)$, which indicated an obviously upregulated expression profile of PURPL in ovarian cancer tissues $(\mathrm{F}=6676.000, \mathrm{P}<0.0001)$. Shown in Figure 5.

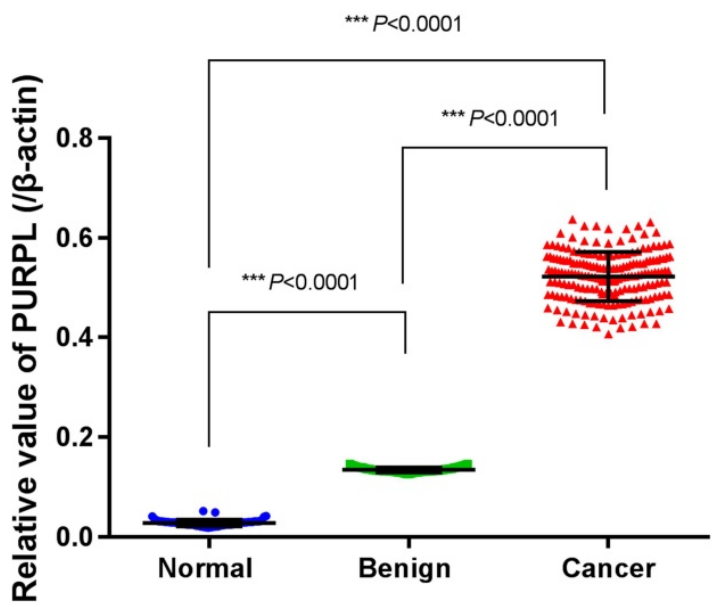

Figure 5. Expressions of PURPL in collected different ovarian tissues measured by real time PCR 


\section{PURPL expressions were related with clinical} stage and lymph node metastasis in EOC

The median relative level of PURPL in 65 cases of epithelial ovarian cancer was $(0.525 \pm 0.006)$, which was used to divide PURPL expression into relatively high group (higher than or equal to the median expression) and relatively low group (lower than the median expression). The expressions of PURPL were significantly higher in the EOC patients with more advanced clinical stage and developed lymph node metastasis (Table 2).

Table 2. The relationships between PURPL expressions in EOC tissues and clinicopathological parameters of EOC (n, \%)

\begin{tabular}{|c|c|c|c|c|c|}
\hline \multirow[t]{2}{*}{ Item } & \multirow[t]{2}{*}{$\mathbf{n}$} & \multicolumn{2}{|l|}{ PURPL } & \multirow[t]{2}{*}{$\chi^{2}$ value } & \multirow[t]{2}{*}{$P$ value } \\
\hline & & High & Low & & \\
\hline \multicolumn{6}{|l|}{ Age } \\
\hline$<50$ year & 31 & $15(48.39)$ & $16(51.61)$ & & \\
\hline$\geq 50$ year & 34 & $18(52.94)$ & $16(47.06)$ & 0.135 & 0.714 \\
\hline \multicolumn{6}{|c|}{ FIGO stage } \\
\hline I-II & 12 & $1(0.08)$ & 11(91.67) & & \\
\hline III-IV & 53 & $31(58.49)$ & $22(41.51)$ & 10.785 & $0.001^{* * *}$ \\
\hline \multicolumn{6}{|c|}{ Histological grade } \\
\hline $\mathrm{G}_{3}$ & 27 & $16(59.26)$ & $11(40.74)$ & & \\
\hline $\mathrm{G}_{1}-\mathrm{G}_{2}$ & 38 & $15(39.47)$ & $23(60.53)$ & 2.477 & 0.116 \\
\hline \multicolumn{6}{|c|}{ Histological classification } \\
\hline Serous & 52 & $32(61.54)$ & $20(38.46)$ & & \\
\hline Mucinous & 13 & $6(46.15)$ & $7(53.85)$ & 1.014 & 0.314 \\
\hline \multicolumn{6}{|l|}{ Ascites } \\
\hline No & 16 & $7(43.75)$ & $9(56.25)$ & & \\
\hline Yes & 49 & $31(63.27)$ & $18(36.73)$ & 1.892 & 0.169 \\
\hline \multicolumn{6}{|c|}{ Lymph node metastasis } \\
\hline No & 33 & $13(39.39)$ & $20(60.61)$ & & \\
\hline Yes & 32 & $21(65.63)$ & $11(34.38)$ & 4.481 & $0.034^{*}$ \\
\hline
\end{tabular}

\section{PURPL was a high risk factor of recurrence and death for the EOC patients}

Binary logistic regression analysis was used to analyze high risk factors of recurrence and death of 65 EOC patients, related factors including PURPL expression, miR-338-3p expression, age, clinical stage, histological grade, histological classification, ascites and lymph node metastasis. Upregulation of PURPL was high risk factor of the recurrence $(\mathrm{P}=0.002$, $\mathrm{OR}=21.482$, 95\%CI: 3.457 94.251) and death ( $\mathrm{P}=0.004$, $\mathrm{OR}=35.643$, 95\%CI: 2.453 84.359) for EOC patients, other risk factors were consistent with our previous results [9].

\section{PURPL expression was negatively related with miR-338-3p expression in different ovarian tissues}

Combining our previous data, we explored the correlation between the expressions of PURPL and miR-338-3p. Analyzed by Spearman correlation test, a significant negative correlation $(\mathrm{r}=-0.970, \mathrm{P}<0.0001)$ between PURPL and miR-338-3p expressions was noted in our collected 105 specimens different ovarian tissues (Figure 6).

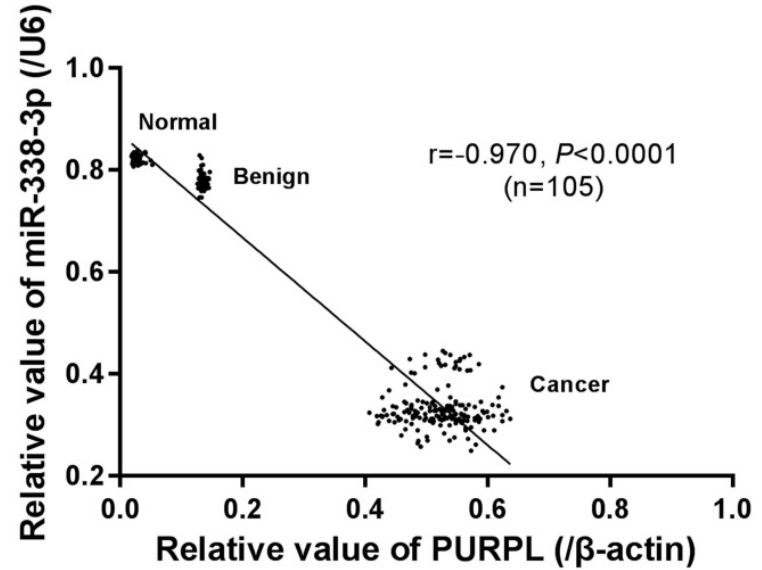

Figure 6. The correlation between PURPL and miR-338-3p expression in collected different ovarian tissues analyzed by Spearman correlation test

\section{Upregulation of PURPL and downregulation of miR-338-3p indicated poor RFS and OS for EOC patients}

We also used the same collected data to analyze the significance of PURPL and miR-338-3p for the prognosis of EOC. The follow-up details of enrolled EOC patients were described in our previous report [9]. The median expression was set to divide sub-expression groups for PURPL and miR-338-3p. The endpoints of the study were death and recurrence. Survival or no recurrence at the end of the follow-up was defined as the censored event, which was censored in statistics. At the end of the follow-up, the RFS rate of the EOC patients with high level of PURPL and low level of miR-338-3p was notably worse than other patients $\left(\chi^{2}=19.410, P=0.0002\right)$, as well as the OS rate $\left(\chi^{2}=17.600, P=0.0005\right)$. Shown in Figure 7.

\section{Discussion}

Exploring the roles of noncoding RNAs in the development and progress of ovarian cancer could provide potential molecular targets and markers for clinical treatment and prognostic monitoring of ovarian cancer. MiRNAs were closely related with the development and progress of human cancers, which had been well proved. The roles of miR-338-3p disregulation in human malignancies were well discussed, such as colorectal carcinoma [17], gastric cancer [18], ovarian epithelial carcinoma [19], breast cancer [20] and lung cancer [21]. The present report was an extension of our previous researches about the relations between miR-338-3p and ovarian cancer. Our reported data indicated miR-338-3p was downregulated in ovarian cancer, and involved in the growth and metastasis of ovarian cancer cells might due to the regulation of cell proliferation and EMT 

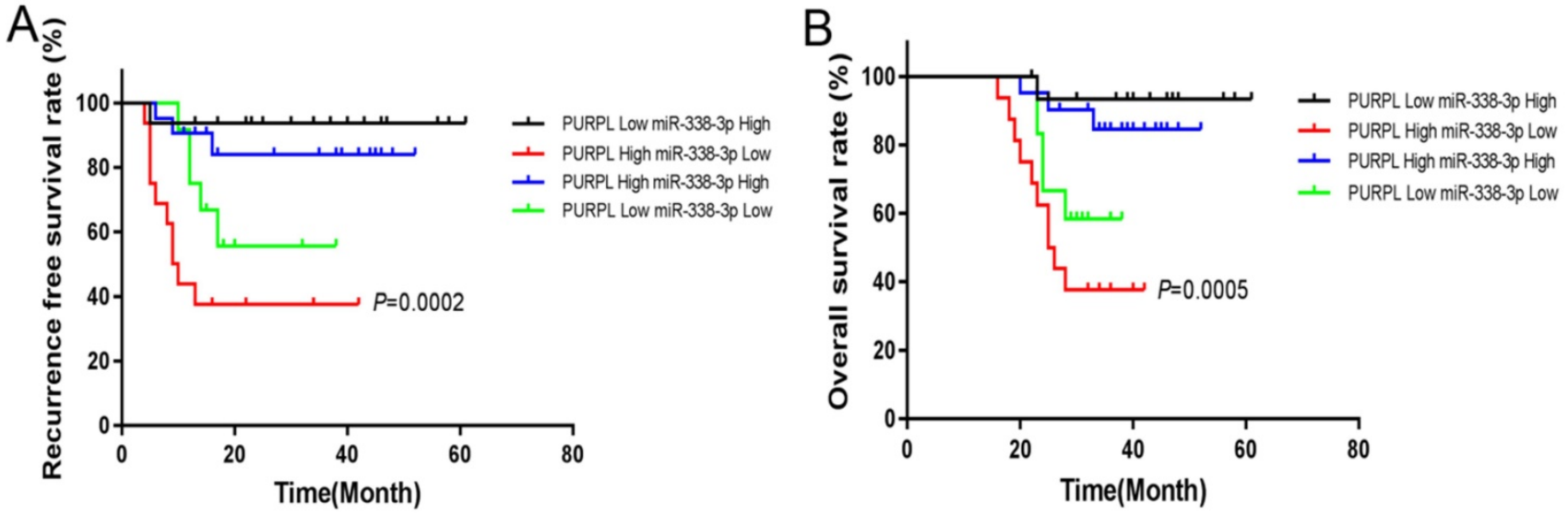

Figure 7. The relations between PURPL and miR-338-3p expressions and RFS and OS of EOC analyzed by Kaplan-Meier analysis

induced by MACC1, Met and its downstream Wnt/ Catenin beta and MEK/ERK signaling pathways [9, 22].

LncRNAs played unignorable roles in human malignant tumors. LncRNAs targeted and sponged miRNAs to mediate downstream genes and signaling pathways were important regulatory mechanisms in human carcinogenesis process [23-25]. In lncRNA/ miRNA regulation network, different lncRNAs could target and sponge miR-338-3p to be implicated in the development and progression of human malignancies [26-29]. In order to analyze the functions of IncRNAs regulated miR-338-3p in ovarian cancer cells, we used biomedical database query to filter candidate upstream lncRNAs for miR-338-3p. Multiple biomedical database cross-queries suggested PURPL could target miR-338-3p.

PURPL is about 1120bp in length, located on human chromosome 5p14.1. PURPL was reported to be involved in the development and progression of colorectal cancer, liver cancer and gastric cancer [30-32]. The roles of PURPL involved in ovarian cancer have never been reported. We dug out database information about PURPL in human malignancy. PURPL was upregulated in several human cancers, including ovarian cancer. Upregulation of PURPL indicated poor RFS and OS for ovarian cancer patients. Moreover, PURPL expression showed a negative expression profile with miR-338-3p expression.

To verify the results queried in biomedical databases, we also collected normal, benign and malignant ovarian tissues to analyze the roles of PURPL in the ovarian cancer. Based on our conserved tissue specimens, expressions of PURPL were detected by real time PCR. Instead of normal ovarian tissues and benign ovarian tumor tissues, obvious upregulations of PURPL were measured in EOC tissues, which suggested abnormal expression of
PURPL might be implicated in the carcinogenesis of ovarian cancer. We also analyzed the relations between PURPL expression and clinicopathological parameters of EOC. Positive relations were noted between PURPL expression and clinical stage and lymph node metastasis of EOC. Furthermore, upregulation of PURPL was a high risk factor for the recurrence and death of EOC patients. These data all indicated upregulation of PURPL might relate to poor prognosis of EOC.

Biomedical database queries suggested PURPL could target miR-338-3p, and PURPL expression shown a negative expression profile with miR-338-3p expression. Therefore, we conducted further analysis about the relation between PURPL and miR-338-3p in collected different ovarian tissues. A significantly negative relation was observed between PURPL expression and miR-338-3p expression in different ovarian tissues. We also analyzed the significances of PURPL and miR-338-3p expressions for the survival of EOC. EOC patients with high level PURPL and low level miR-338-3p presented worse RFS and OS rate than other EOC patients. These data indicated that disregulations of PURPL and miR-338-3p were implicated in the development and progression of EOC, and could serve as prognosis biomarkers for EOC.

\section{Conclusions}

According to biomedical databases query and collected cohort tissue analysis, our present data suggested that PURPL was upregulated in EOC, PURPL expression was negatively related to miR338-3p, and disregulations of PURPL and miR-338-3p presented poor prognosis of EOC.

\section{Abbreviations}

lncRNAs: long noncoding RNAs; PURPL: p53 upregulated regulator of p53 levels; miR-338-3p: 
microRNA-338-3p; EOC: epithelial ovarian cancer; RFS: recurrence free survival; OS: overall survival; miRNAs: micro noncoding RNAs.

\section{Acknowledgments}

We thank the Clinical Medicine Key Disciplines Laboratory of Henan Province for assistance with experiments. Our work was supported by the Scientific and technological projects of Henan Province (202102310392), and in part by the Youth Innovation Fund Project of the First Affiliated Hospital of Zhengzhou University (201418).

\section{Competing Interests}

The authors have declared that no competing interest exists.

\section{References}

1. Siegel RL, Miller KD, Fuchs HE, Jemal A. Cancer Statistics, 2021. CA Cancer J Clin. 2021;71(1):7-33.

2. Adelman K, Egan E. NON-CODING RNA More uses for genomic junk. Nature. 2017;543(7644):183-5

3. Anastasiadou E, Jacob LS, Slack FJ. Non-coding RNA networks in cancer. Nat Rev Cancer. 2018;18(1):5-18.

4. Ghildiyal M, Zamore PD. Small silencing RNAs: an expanding universe. Nat Rev Genet. 2009;10(2):94-108.

5. Iorio MV, Croce CM. microRNA involvement in human cancer. Carcinogenesis. 2012;33(6):1126-33.

6. Yarmishyn AA, Kurochkin IV . Long noncoding RNAs: a potential novel class of cancer biomarkers. Front Genet. 2015;6:145

7. Renganathan A, Felley-Bosco E. Long Noncoding RNAs in Cancer and Therapeutic Potential. Adv Exp Med Biol. 2017,1008:199-222.

8. Dhamija S, Diederichs S. From junk to master regulators of invasion: IncRNA functions in migration, EMT and metastasis. Int J Cancer. 2016;139(2):269-80.

9. Zhang R, Shi H, Ren F, Liu Z, Ji P, Zhang W, et al. Down-regulation of MiR-338-3p and Up-regulation of MACC1 Indicated Poor Prognosis of Epithelial Ovarian Cancer Patients. J Cancer. 2019;10(6):1385-92.

10. Moridi H, Karimi J, Tavilani H, Khodadadi I, Emami Razavi AN Overexpression of PURPL and downregulation of NONHSAT062994 as potential biomarkers in gastric cancer. Life Sci. 2019;237:116904.

11. Jun-Hao Li, Shun Liu, Hui Zhou, Liang-Hu Ou, Jian-Hua Yang. StarBase v2.0: decoding miRNA-ceRNA, miRNA-ncRNA and protein-RNA interaction networks from large-scale CLIP-Seq data. Nucleic Acids Res. 2014;42(D):D92-7.

12. Maria D Paraskevopoulou, Ioannis S Vlachos, Dimitra Karagkouni, Georgios Georgakilas, Ilias Kanellos, Thanasis Vergoulis, et al. DIANA-LncBase v2: indexing microRNA targets on non-coding transcripts. Nucleic Acids Res. 2016;44(D1):D231-8.

13. Gao JJ, Aksoy BA, Dogrusoz U, Dresdner G, Gross B, Sumer SO, et al. Integrative Analysis of Complex Cancer Genomics and Clinical Profiles Using the cBioPortal. Sci Signal. 2013;6(269):pl1.

14. Ya-Ru Miao, Wei Liu, Qiong Zhang, An-Yuan Guo. IncRNASNP2: an updated database of functional SNPs and mutations in human and mouse lncRNAs. Nucleic Acids Res. 2018;46(D1):D276-80.

15. Zefang Tang, Chenwei Li, Boxi Kang, Ge Gao, Cheng Li, Zemin Zhang. GEPIA: a web server for cancer and normal gene expression profiling and interactive analyses. Nucleic Acids Res. 2017;45(W1):W98-102.

16. Gyorffy B, Lanczky A, Eklund AC, Denkert C, Budczies J, Li Q et al. An online survival analysis tool to rapidly assess the effect of 22,277 genes on breast cancer prognosis using microarray data of 1809 patients. Breast Cancer Res Treatment. 2010;123(3):725-31.

17. Sun K, Su G, Deng H, Dong J, Lei S, Li G. Relationship between miRNA-338-3p expression and progression and prognosis of human colorectal carcinoma. Chin Med J (Engl). 2014;127(10):1884-90.

18. Liu S, Suo J, Wang C, Sun $\mathrm{X}$, Wang D, He L, et al Downregulation of tissue miR-338-3p predicts unfavorable prognosis of gastric cancer. Cancer Biomark. 2017;21(1):117-22.

19. Wen C, Liu X, Ma H, Zhang W, Li H. miR-338-3p suppresses tumor growth of ovarian epithelial carcinoma by targeting Runx2. Int J Oncol. 2015;46(5):2277-85

20. Jin $\mathrm{Y}$, Zhao $\mathrm{M}$, Xie $\mathrm{Q}$, Zhang $\mathrm{H}$, Wang $\mathrm{Q}$, Ma Q. MicroRNA-338-3p functions as tumor suppressor in breast cancer by targeting SOX4. Int J Oncol. 2015;47(4):1594-602.
21. Li Y, Chen P, Zu L, Liu B, Wang M, Zhou Q. MicroRNA-338-3p suppresses metastasis of lung cancer cells by targeting the EMT regulator Sox4. Am J Cancer Res. 2016;6(2):127-40.

22. Zhang R, Shi H, Ren F, Feng W, Cao Y, Li G, et al. MicroRNA-338-3p suppresses ovarian cancer cells growth and metastasis: implication of Wnt/catenin beta and MEK/ERK signaling pathways. J Exp Clin Cancer Res. 2019;38(1):494.

23. Goodall GJ, Wickramasinghe VO. RNA in cancer. Nat Rev Cancer. 2021;21(1):22-36

24. Chen S, Shen X. Long noncoding RNAs: functions and mechanisms in colon cancer. Mol Cancer. 2020;19(1):167.

25. Md Yusof K, Rosli R, Abdullah M, Avery-Kiejda KA. The Roles of Non-Coding RNAs in Tumor-Associated Lymphangiogenesis. Cancers (Basel). 2020;12(11):3290.

26. $\mathrm{Hu} \mathrm{CH}$, Yang $\mathrm{XJ}$, Yu L, Wang $\mathrm{LY}$, Zhao XC, Han $\mathrm{CH}$. Long non-coding RNA LINC00173 serves as sponge for miR-338-3p to promote prostate cancer progression via regulating Rab25. Eur Rev Med Pharmacol Sci. 2020;24(18):9290-302

27. Song W, Wang K, Yang X, Dai W, Fan Z. Long non-coding RNA BANCR mediates esophageal squamous cell carcinoma progression by regulating the IGF1R/Raf/MEK/ERK pathway via miR-338-3p. Int $\mathrm{J}$ Mol Med. 2020;46(4):1377-88.

28. Lu H, Zhang Q, Sun Y, Wu D, Liu L. LINC00689 induces gastric cancer progression via modulating the miR-338-3p/HOXA3 axis. J Gene Med. 2020;22(12):e3275

29. Ji D, Hu G, Zhang X, Yu T, Yang J. Long non-coding RNA DSCAM-AS1 accelerates the progression of hepatocellular carcinoma via sponging miR-338-3p. Am J Transl Res. 2019;11(7):4290-302.

30. Li XL, Subramanian M, Jones MF, Chaudhary R, Singh DK, Zong X, et al. Long Noncoding RNA PURPL Suppresses Basal p53 Levels and Promotes Tumorigenicity in Colorectal Cancer. Cell Rep. 2017;20(10):2408-23.

31. Fu X, Wang $Y$, Wu G, Zhang $W, X u$ S, Wang $W$. Long noncoding RNA PURPL promotes cell proliferation in liver cancer by regulating p53. Mol Med Rep. $2019 ; 19(6): 4998-5006$.

32. Xu F, Ji Z, He L, Chen M, Chen H, Feng $Q$, et al. Downregulation of LINC01021 by curcumin analog Da0324 inhibits gastric cancer progression through activation of P53. Am J Transl Res. 2020 ;12(7):3429-44. 